\title{
Impact of postcolectomy adhesion-related complications on healthcare utilization
}

This article was published in the following Dove Press journal:

ClinicoEconomics and Outcomes Research

\section{Katherine Etter ${ }^{\prime}$ \\ Nadia Sutton ${ }^{2}$ \\ David $\mathrm{Wei}^{3}$ \\ Andrew Yoo $^{3}$}

'Medical Devices-Epidemiology, Johnson and Johnson, Raynham, MA 02767, USA; ${ }^{2}$ Ethicon HEMA, Somerville, NJ 08876, USA; ${ }^{3}$ Medical Devices-Epidemiology, Johnson and Johnson, New Brunswick, NJ 0890I, USA
Correspondence: Andrew Yoo Medical Devices-Epidemiology, Johnson and Johnson, 410 George Street, New Brunswick, NJ 0890I, USA

$\mathrm{Tel}+\mathrm{I} 732524$ I354

Email ayoo@its.jnj.com
Objective: The objective of this study was to explore adhesion-related complications (ARCs) within 1 year after colectomy.

Methods: Using Truven MarketScan ${ }^{\circledR}$ Commercial and Medicare databases, the first inpatient colectomies during 2009-2013 (index) were identified: left, right, partial, transverse, or total. One-year continuous enrollment was required pre and postindex. Only the first inpatient rehospitalization event was analyzed. ARC was defined as the subset of rehospitalizations with a diagnosis of ileus, small bowel obstruction, or postindex adhesiolysis. ARC and non-ARC events were evaluated descriptively, including time to ARC, length of stay (LOS), and total hospitalization reimbursement (2015 US dollars [2015 USD]). Patient, provider, and procedure factors associated with ARC were explored using logistic regression models.

Results: A total of 64,532 colectomies were identified: left (39.2\%), right (34.9\%), partial $(20.0 \%)$, transverse (2.3\%), and total (3.6\%). Surgical approach was classified as open $(60.1 \%)$ and laparoscopic (39.9\%). All-cause first inpatient readmission incidence within 1 year was $24.7 \%$, and ARC incidence was $5.7 \%$ in all patients or $23.2 \%$ in all first readmissions. ARC had statistically higher resource utilization compared to non-ARC with respective mean (SD) time to event (130 [102] and 137 [106] days), mean (SD) LOS (7.2 [8.0] and 5.2 [6.8] days), and mean (SD) total reimbursement $(\$ 29,802[\$ 43,037]$ and $\$ 22,476[\$ 36,130])$. ARC risk factors included (OR [95\% CI]) resection type (total vs right, 3.78 [3.27-4.36]), left vs right (1.69 [1.53-1.86]), adhesiolysis (2.45 [1.42-4.23]), computerized tomography (1.79 [1.65-1.95]), surgical indication: inflammatory bowel disease vs cancer (1.69 [1.43-1.99]), and multiple abdominal procedures (1.38 [1.29-1.49]). Laparoscopic approach was protective (0.42 [0.39-0.46]).

Conclusion: ARCs were associated with almost one-fourth of all first rehospitalizations within the first year after colectomy and were associated with substantial healthcare utilization. Risk factors included increased index colectomy complexity, while the laparoscopic approach was protective. Future research is needed to better identify high-risk patients and allow for appropriate economic and clinical risk adjustment of outcomes.

Keywords: bowel obstruction, ileus, rehospitalization

\section{Introduction}

Adhesions are caused by intra-abdominal scar tissue that can form within a short period in patients who undergo abdominal or pelvic surgery. ${ }^{1}$ During the inflammatory process following the tissue trauma, macrophages, fibroblasts, and a fibrin matrix flood the wound area, thus leading to the formation of an adhesion. ${ }^{2}$ More than 400,000 adhesiolysis (the surgical dissection of adhesions) procedures are performed yearly in the US with an estimated economic impact of $\sim \$ 2.3$ billion. ${ }^{3}$ Adhesions can lead 
to acute life-threatening postsurgical complications such as small bowel obstructions (SBOs) and ileus. Other more chronic manifestations include infertility and pain. ${ }^{1,2}$ These complications may lead to significantly lower quality of life in patients, longer hospital stays, increased hospitalization, and higher costs. ${ }^{3-5}$ Of all types of abdominal surgery, open colorectal surgery was reported to result in the highest rate of adhesion-related hospital readmissions. ${ }^{4}$

In the US, procedures for SBO are among the top 10 of emergency general surgeries that count for as much as $80 \%$ of morbidity and death related to emergency surgery. ${ }^{6}$ Adhesions have been listed as the most common cause of SBO occurring in as much as $56 \%-75 \%$ of all cases of SBO. ${ }^{7-11}$ Patient-related factors such as age and gender as well as surgery-related factors such as previous abdominal surgery, anatomy and extent of colorectal resection, emergency surgery, and inflammatory conditions can affect the incidence of adhesion-related SBO. ${ }^{9}$ Furthermore, the incidence of adhesion-related SBO is very high in patients who had rectal surgery, especially pelvic and ileoanal pouch surgeries. ${ }^{5-12}$ As much as $23 \%$ of patients are readmitted within 90 days of their original colorectal surgery. ${ }^{10}$ However, these estimates are mostly derived from small case series and older retrospective studies.

Remarkably, there is a lack of recent data related to the overall burden of adhesion-related complications (ARCs) after colectomies. Estimates related to incidence, healthcare utilization and costs of ARC after colectomies may aid in appropriate patient and procedural risk adjustment that may inform reimbursement. Additionally, this can also better inform patients and surgeons regarding the impact of this clinical outcome. Hence, the objective of this study was to explore the first rehospitalization within 1 year after colon resections. This included ARC incidence, time to event, risk factors, and healthcare utilization for inpatient hospitalizations and costs. We also evaluated non-ARC first rehospitalization to provide context for ARC readmissions.

\section{Methods}

\section{Data source}

This retrospective cohort study used US administrative claims data from the Truven Health MarketScan ${ }^{\circledR}$ (Truven Health Analytics, Ann Arbor, MI, USA) Commercial and Medicare Supplemental databases. The databases contain inpatient medical, outpatient medical, outpatient prescription administrative claims data, and enrollment records of individuals with employer-sponsored primary or Medicare supplemental insurance. Data for $>70$ million individuals collected from $>300$ large self-insured US employers and $>25$ US health plans are in these databases. Information for primary insured individuals, a spouse, or a dependent of a spouse who are under the age of 65 years is contained in the Commercial Claims and Encounters database. The Medicare Supplemental and Coordination of Benefits database includes information for individuals who are Medicare eligible and have a supplemental insurance paid for by their current or former employer. The data were previously collected, de-identified, and compliant with the privacy regulations of the Health Insurance Portability and Accountability Act. Therefore, institutional review board's approval and written informed consent were not required for this study.

\section{Patient selection}

The study population comprised all patients who underwent an inpatient colectomy between 2009 and 2013 in the Truven Health MarketScan ${ }^{\circledR}$ database. ICD ninth revision (ICD-9) procedure codes were utilized to identify the first colectomy event. Colectomy resections were classified by anatomy: left (hemicolectomy/sigmoidectomy), right (cecectomy/hemicolectomy), partial, transverse, or total colectomy.

\section{Inclusion and exclusion criteria}

The date of patient's first inpatient colectomy during the study period was assigned as the index procedure date (index). To be included in the study, 1-year continuous enrollment was required before (preindex) and after (postindex) the index colectomy procedure. Patients were excluded if there was an evidence of adhesions or an ARC in the preindex period.

\section{Study outcomes}

All postindex first rehospitalization events in the postindex period were analyzed. ARC events were defined as the subset of first rehospitalizations with either ICD-9 diagnosis codes for SBO or ileus or an ICD-9 procedure code indicating adhesiolysis. Incidence and time to first rehospitalization were calculated. Finally, risk factors for having an ARC were identified.

Healthcare utilization outcomes for both ARC and nonARC included length of stay (LOS), total hospital reimbursement inflated to 2015 US dollars (2015 USD), and Medicare Severity Diagnosis Related Groupings (MS-DRG) category: major complication or comorbidity (MCC), complication or comorbidity (CC), or without MCC or CC. Additionally, the rate of computed tomography $(\mathrm{CT})$ scans any time postindex was calculated. 


\section{Study covariates}

Various patient- and procedure-related covariates were included in the study. All diagnoses for surgical indication and comorbidities were identified with ICD-9 diagnosis codes. Additionally, ICD-9 procedure codes were utilized to identify procedural covariates that included adhesiolysis and concomitant abdominal procedures (refer Table S1 for a list of ICD-9 codes). CT imaging at index was identified with Current Procedural Terminology (CPT-4) and MarketScan reimbursement codes.

Patient characteristics included demographics in the 1 year prior to surgery such as age and gender and specific patient comorbidities from the Elixhauser comorbidity categories (cardiac arrhythmia, congestive heart failure, valvular disease, peripheral vascular disease, myocardial infarction, cerebrovascular disease, pulmonary circulation disorder, obesity, complicated diabetes, uncomplicated diabetes, hypothyroidism, complicated hypertension, uncomplicated hypertension, depression, alcohol abuse, and drug abuse). ${ }^{13}$

Index procedure characteristics included in the study were year of surgery, type of anatomic colon resection (left, right, partial, transverse, and total), surgical approach (laparoscopic vs open), indication for surgery (diverticulitis, diverticulosis, inflammatory bowel disease [IBD; included both Crohn's disease and ulcerative colitis], cancer, and others), adhesiolysis, the number of concomitant abdominal procedures performed, robotic-assisted procedure (ICD-9 code 17.4X), and whether a CT scan was performed.

\section{Statistical analyses}

Descriptive analyses were performed for all study variables and included mean (SD) values for continuous variables and frequency distributions for categorical variables. These were presented for patients with and without a readmission during the postindex period. Patients with a readmission were further divided into all-cause readmissions, ARC, and non-ARC groups.

A bivariate analysis comparing differences between ARC and non-ARC resource utilization was performed using Wilcoxon signed-rank test for non-normally distributed continuous variables and chi-squared test for proportions. Patient and procedure factors associated with ARC were explored in a multivariable logistic regression model evaluating the odds of having an ARC within 1 year of colon resection. Statistical significance was set a priori at $P<0.05$ (two sided). All analyses were conducted using SAS for Windows, Version 9.4 (SAS Institute Inc., Cary, NC, USA).

\section{Results \\ Descriptive patient and procedure characteristics}

A total of 64,532 patients underwent inpatient colectomy (resection type: left [39.2\%], right [34.9\%], partial [20.0\%], transverse [2.3\%], and total [3.6\%]). Open surgery was the most common surgical approach $(60.1 \%)$. A majority $(70.1 \%)$ of the patients had commercial insurance. Female and male patients were balanced in the sample with $51.7 \%$ female patients. The mean age of all patients was 58.4 years ( $\mathrm{SD}=13.7)$. Cancer $(31.2 \%)$ was the most common specific indication of the colorectal surgery followed by diverticulitis (27.7\%), IBD (3.3\%), and diverticulosis (2.7\%). Almost one-third $(29.9 \%)$ of patients had a concomitant procedure. A small number of patients had adhesiolysis at index $(\mathrm{n}=92)$. Hypertension (36.2\%), fluid and electrolyte disorders (12.4\%), and diabetes (11.7\%) were the most common comorbidities. Table 1 represents the patient demographics, and Table 2 represents the procedural characteristics of the entire study population.

\section{Incidence of ARC and all-cause inpatient readmission rates}

All-cause (ARC and non-ARC) first inpatient readmissions within 1 year of index was $24.7 \%(n=15,933)$. The ARC accounted for $23.2 \%(n=3,704)$ of all-cause first inpatient readmissions within 1 year of index. Table 3 shows a breakdown of the ARC incidence by resection type: total colectomy $(19.3 \%)$ had the highest incidence, followed by left-sided colectomy (6.4\%), and right-sided colectomy (4.1\%) had the lowest incidence. ARC as a proportion of all first rehospitalization events by resection type ranged from $35.4 \%$ for total colectomy to $14.7 \%$ for partial colectomy.

Figure 1 illustrates the yearly trends in rehospitalization, ARC, and non-ARC incidence. The breakdown for individual ARC components is also given. Within the ARC cohort, 9.7\% of patients had more than one event (adhesiolysis, ileus, or SBO). Accounting for nonmutually exclusive ARC categories, $46.5 \%$ of patients had a SBO diagnosis, $34.4 \%$ had an ileus diagnosis, and 29.2\% underwent adhesiolysis. Among patients who had an ARC-related readmission, the mean time to $\mathrm{ARC}$ event was 130 days ( $\mathrm{SD}=102$, median=112 days).

Table 1 describes patient demographics, and Table 2 presents the procedural characteristics associated with patients with and without ARC. As anticipated, patients without readmission events had lower comorbidities as indicated by lower Elixhauser comorbidity scores. ARC patients were 
Table I Patient and procedure characteristics stratified by readmission status

\begin{tabular}{|c|c|c|c|c|c|c|c|c|}
\hline & \multicolumn{2}{|l|}{ All } & \multicolumn{2}{|c|}{$\begin{array}{l}\text { No } \\
\text { rehospitalization }\end{array}$} & \multicolumn{2}{|c|}{$\begin{array}{l}\text { Non-ARC } \\
\text { rehospitalization }\end{array}$} & \multicolumn{2}{|c|}{$\begin{array}{l}\text { ARC } \\
\text { rehospitalization }\end{array}$} \\
\hline & $\bar{n}$ & $\%$ & $\bar{n}$ & $\%$ & $\bar{n}$ & $\%$ & $\bar{n}$ & $\%$ \\
\hline Number of patients & 64,532 & 100 & 48,599 & 100 & 12,229 & 100 & 3,704 & 100 \\
\hline \multicolumn{9}{|l|}{ Gender } \\
\hline Female & 33,355 & 51.7 & 25,101 & 51.7 & 6,331 & 51.8 & 1,923 & 51.9 \\
\hline Male & 31,177 & 48.3 & 23,498 & 48.4 & 5,898 & 48.2 & $|, 78|$ & 48.1 \\
\hline \multicolumn{9}{|l|}{ Age (years) } \\
\hline $18-44$ & 9,065 & 14.1 & 6,655 & 13.7 & 1,685 & 13.8 & 725 & 19.6 \\
\hline $45-54$ & 16,276 & 25.2 & 12,732 & 26.2 & 2,571 & 21.0 & 973 & 26.3 \\
\hline $55-64$ & 20,115 & 31.2 & $15,2 \mid 7$ & 31.3 & 3,704 & 30.3 & $\mathrm{I}, 194$ & 32.2 \\
\hline $65-74$ & 9,692 & 15.0 & 7,285 & 15.0 & 1,938 & 15.9 & 469 & 12.7 \\
\hline $75+$ & 9,384 & 14.5 & 6,710 & 13.8 & 2,331 & 19.1 & 343 & 9.3 \\
\hline \multicolumn{9}{|l|}{ Payer } \\
\hline Medicare & 19,324 & 29.9 & 14,162 & 70.9 & 4,333 & 64.6 & 829 & 22.4 \\
\hline Commercial & 45,208 & 70.1 & 34,437 & 29.1 & 7,896 & 35.4 & 2,875 & 77.6 \\
\hline \multicolumn{9}{|l|}{ Elixhauser score } \\
\hline 0 & 13,143 & 20.4 & 10,413 & 21.4 & 1,952 & 16.0 & 778 & 21.0 \\
\hline $\mathrm{I}-2$ & 31,229 & 48.4 & 24,263 & 49.9 & 5,285 & 43.2 & $1,68 \mid$ & 45.4 \\
\hline $3-4$ & 14,196 & 22.0 & 10,226 & 21.0 & $3,|4|$ & 25.7 & 829 & 22.4 \\
\hline $5+$ & 5,964 & 9.2 & 3,697 & 7.6 & $|, 85|$ & 15.1 & 416 & 11.2 \\
\hline \multicolumn{9}{|l|}{ Elixhauser comorbidities } \\
\hline Solid tumor without metastasis & 25,467 & 39.5 & 19,140 & 39.4 & 5,082 & 41.6 & 1,245 & 33.6 \\
\hline Hypertension uncomplicated & 23,383 & 36.2 & 17,565 & 36.1 & 4,650 & 38.0 & 1,168 & 31.5 \\
\hline Fluid and electrolyte disorders & 7,997 & 12.4 & 5,114 & 10.5 & 2,147 & 17.6 & 736 & 19.9 \\
\hline Diabetes uncomplicated & 7,525 & 11.7 & 5,404 & 11.1 & 1,736 & 14.2 & 385 & 10.4 \\
\hline Chronic pulmonary disease & 6,876 & 10.7 & 4,824 & 9.9 & 1,632 & 13.4 & 420 & 11.3 \\
\hline Cardiac arrhythmia & 6,673 & 10.3 & 4,466 & 9.2 & I,792 & 14.7 & 415 & 11.2 \\
\hline Metastatic cancer & 6,098 & 9.5 & 4,049 & 8.3 & 1,626 & 13.3 & 423 & 11.4 \\
\hline Obesity & 5,407 & 8.4 & 4,061 & 8.4 & 1,065 & 8.7 & 281 & 7.6 \\
\hline Hypothyroidism & 5,055 & 7.8 & 3,785 & 7.8 & 989 & 8.1 & 281 & 7.6 \\
\hline Liver disease & 4,305 & 6.7 & 3,207 & 6.6 & 860 & 7.0 & 238 & 6.4 \\
\hline Depression & $3,77 \mid$ & 5.8 & 2,702 & 5.6 & 814 & 6.7 & 255 & 6.9 \\
\hline Deficiency anemia & 3,630 & 5.6 & 2,562 & 5.3 & 856 & 7.0 & 212 & 5.7 \\
\hline Weight loss & 2,892 & 4.5 & 1,667 & 3.4 & 884 & 7.2 & 341 & 9.2 \\
\hline Peripheral vascular disorders & 2,677 & 4.2 & I,7I7 & 3.5 & 769 & 6.3 & 191 & 5.2 \\
\hline Congestive heart failure & 2,235 & 3.5 & $\mathrm{I}, 30 \mathrm{I}$ & 2.7 & 802 & 6.6 & 132 & 3.6 \\
\hline Valvular disease & 2,101 & 3.3 & $\mathrm{I}, 443$ & 3.0 & 546 & 4.5 & 112 & 3.0 \\
\hline Renal failure & $\mathrm{I}, 778$ & 2.8 & I,037 & 2.1 & 609 & 5.0 & 132 & 3.6 \\
\hline Hypertension complicated & 1,682 & 2.6 & $\mathrm{I}, 040$ & 2.1 & 523 & 4.3 & 119 & 3.2 \\
\hline Blood loss anemia & 1,536 & 2.4 & $\mathrm{I}, 038$ & 2.1 & 386 & 3.2 & 112 & 3.0 \\
\hline Other neurological disorders & 1,283 & 2.0 & 781 & 1.6 & 397 & 3.3 & 105 & 2.8 \\
\hline Rheumatoid arthritis/collagen & 1,239 & 1.9 & 844 & 1.7 & 316 & 2.6 & 79 & 2.1 \\
\hline Coagulopathy & 1,187 & 1.8 & 716 & 1.5 & 372 & 3.0 & 99 & 2.7 \\
\hline Pulmonary circulation disorders & 884 & 1.4 & 529 & 1.1 & 289 & 2.4 & 66 & 1.8 \\
\hline Diabetes complicated & 925 & 1.4 & 533 & I.I & 340 & 2.8 & 52 & 1.4 \\
\hline Alcohol abuse & 820 & 1.3 & 539 & I.I & 219 & 1.8 & 62 & 1.7 \\
\hline Peptic ulcer disease excluding bleeding & 549 & 0.9 & 385 & 0.8 & 124 & 1.0 & 40 & 1.1 \\
\hline Lymphoma & 514 & 0.8 & 318 & 0.7 & 162 & 1.3 & 34 & 0.9 \\
\hline Drug abuse & $44 I$ & 0.7 & 267 & 0.6 & 132 & I.I & 42 & I.I \\
\hline Psychoses & 240 & 0.4 & 137 & 0.3 & 83 & 0.7 & 20 & 0.5 \\
\hline Paralysis & 184 & 0.3 & 82 & 0.2 & 90 & 0.7 & 12 & 0.3 \\
\hline AIDS/HIV & 70 & 0.1 & 41 & 0.1 & 22 & 0.2 & 7 & 0.2 \\
\hline
\end{tabular}

Abbreviation: ARC, adhesion-related complication. 
Table 2 Index procedure characteristics stratified by rehospitalization status

\begin{tabular}{|c|c|c|c|c|c|c|c|c|}
\hline & \multicolumn{2}{|l|}{ All } & \multicolumn{2}{|c|}{$\begin{array}{l}\text { No } \\
\text { rehospitalization }\end{array}$} & \multicolumn{2}{|c|}{$\begin{array}{l}\text { Non-ARC } \\
\text { rehospitalization }\end{array}$} & \multicolumn{2}{|c|}{$\begin{array}{l}\text { ARC } \\
\text { rehospitalization }\end{array}$} \\
\hline & $\mathbf{n}$ & $\%$ & $\mathbf{n}$ & $\%$ & $\mathbf{n}$ & $\%$ & $\mathbf{n}$ & $\%$ \\
\hline Number of patients & 64,532 & 100 & 48,599 & 100 & 12,229 & 100 & 3,704 & 100 \\
\hline \multicolumn{9}{|l|}{ Indication_for surgery } \\
\hline Other & 22,651 & 35.1 & 17,253 & 35.5 & 4,048 & 33.1 & 1,319 & 35.6 \\
\hline Cancer & 20,134 & 31.2 & $|5,2| 1$ & 31.3 & 4,023 & 32.9 & 919 & 24.8 \\
\hline Diverticulitis & 17,875 & 27.7 & 13,705 & 28.2 & 3,167 & 25.9 & 1,026 & 27.7 \\
\hline IDB & 2,130 & 3.3 & 1,118 & 2.3 & 673 & 5.5 & 348 & 9.4 \\
\hline Diverticulosis & $\mathrm{I}, 742$ & 2.7 & $|, 36|$ & 2.8 & 318 & 2.6 & 93 & 2.5 \\
\hline \multicolumn{9}{|l|}{ Colon resection type } \\
\hline Left & 25,292 & 39.2 & $|8,7| \mid$ & 38.5 & 4,970 & 40.6 & 1,611 & 43.5 \\
\hline Right & 22,530 & 34.9 & 17,843 & 20.3 & 3,769 & 19.3 & 918 & 24.8 \\
\hline Partial & 12,874 & 20.0 & 9,858 & 36.7 & 2,365 & 30.8 & 651 & 17.6 \\
\hline Total & 2,328 & 3.6 & 1,060 & 2.2 & 819 & 6.7 & 449 & 12.1 \\
\hline Transverse & $\mathrm{I}, 508$ & 2.3 & 1,127 & 2.3 & 306 & 2.5 & 75 & 2.0 \\
\hline \multicolumn{9}{|l|}{ Surgical approach } \\
\hline Open & 38,769 & 60.1 & 27,142 & 55.9 & 8,730 & 71.4 & 2,897 & 78.2 \\
\hline Laparoscopic & 25,763 & 39.9 & 21,457 & 44.2 & 3,499 & 28.6 & 807 & 21.8 \\
\hline \multicolumn{9}{|l|}{ Year of procedure } \\
\hline 2009 & 11,562 & 17.9 & 8,637 & 17.8 & 2,276 & 18.6 & 649 & 17.5 \\
\hline 2010 & 13,970 & 21.7 & 10,450 & 21.5 & 2,700 & 22.1 & 820 & 22.1 \\
\hline 2011 & 15,120 & 23.4 & $|1,39|$ & 23.4 & $2,84 I$ & 23.2 & 888 & 24.0 \\
\hline 2012 & 11,912 & 18.5 & 9,006 & 18.5 & 2,234 & 18.3 & 672 & 18.1 \\
\hline 2013 & 11,968 & 18.6 & 9,115 & 18.8 & 2,178 & 17.8 & 675 & 18.2 \\
\hline Adhesiolysis at index & 92 & 0.1 & 51 & 0.1 & 24 & 0.2 & 17 & 0.5 \\
\hline \multicolumn{9}{|c|}{ Number of procedures at index } \\
\hline 1 & 45,228 & 70.1 & 34,707 & 71.4 & 8,195 & 67.0 & 2,326 & 62.8 \\
\hline $2+$ & 19,304 & 29.9 & 13,892 & 28.7 & 4,034 & 32.9 & I,378 & 37.3 \\
\hline
\end{tabular}

Abbreviations: ARC, adhesion-related complication; IDB, inflammatory bowel disease.

Table 3 Breakdown of ARC components

\begin{tabular}{|c|c|c|c|c|}
\hline & $\mathbf{n}$ & $\begin{array}{l}\text { Proportion } \\
\text { of ARC (\%) }\end{array}$ & Incidence (\%) & $\begin{array}{l}\text { Proportion of all first } \\
\text { rehospitalizations (\%) }\end{array}$ \\
\hline \multicolumn{5}{|l|}{ Resection type } \\
\hline Left & $\mathrm{I}, 6 \mathrm{II}$ & 43.5 & 6.4 & 24.5 \\
\hline Right & 918 & 24.8 & 4.1 & 28.0 \\
\hline Partial & 651 & 17.6 & 5.1 & 14.7 \\
\hline Total & 449 & 12.1 & 19.3 & 35.4 \\
\hline Transverse & 75 & 2.0 & 5.0 & 19.7 \\
\hline \multicolumn{5}{|c|}{ ARC individual component breakdown } \\
\hline All ARC & 3,704 & 100.0 & 5.7 & 23.2 \\
\hline SBO only & $\mathrm{I}, 45 \mathrm{I}$ & 39.2 & 2.2 & 9.1 \\
\hline Ileus only & 979 & 26.4 & 1.5 & 6.1 \\
\hline Adhesiolysis only & 914 & 24.7 & 1.4 & 5.7 \\
\hline Adhesiolysis+SBO & 191 & 5.2 & 0.3 & 1.2 \\
\hline Adhesiolysis+ileus & 87 & 2.3 & 0.1 & 0.5 \\
\hline lleus + SBO & 64 & 1.7 & 0.1 & 0.4 \\
\hline Adhesiolysis+ileus+SBO & 18 & 0.5 & 0.0 & 0.1 \\
\hline \multicolumn{5}{|c|}{ Nonmutually exclusive categories } \\
\hline SBO & $\mathrm{I}, 724$ & - & 2.7 & 10.8 \\
\hline lleus & 1,275 & - & 2.0 & 8.0 \\
\hline Adhesiolysis & $\mathrm{I}, 083$ & - & 1.7 & 6.8 \\
\hline
\end{tabular}

Abbreviations: ARC, adhesion-related complication; SBO, small bowel obstruction. 
younger than non-ARC patients, which was also reflected in the higher proportion of ARC patients with commercial insurance (77.6\%) compared to non-ARC (35.4\%) patients. ARC patients had higher rates of IBD (9.4\%) and lower rates of cancer $(24.8 \%)$ compared to non-ARC patients $(5.5 \%$ and $32.9 \%$, respectively). Procedural differences (Table 2) included ARC patients having more total colectomy (12.1\%) and right colectomy (24.8\%) compared to non-ARC patients (6.7\% and $19.3 \%$, respectively). Partial colectomy was higher in the non-ARC population (30.8\%) vs ARC population (17.6\%). The surgical approach at index colon resections varied with patients without a readmission event having a higher proportion of laparoscopic surgery $(44.2 \%)$

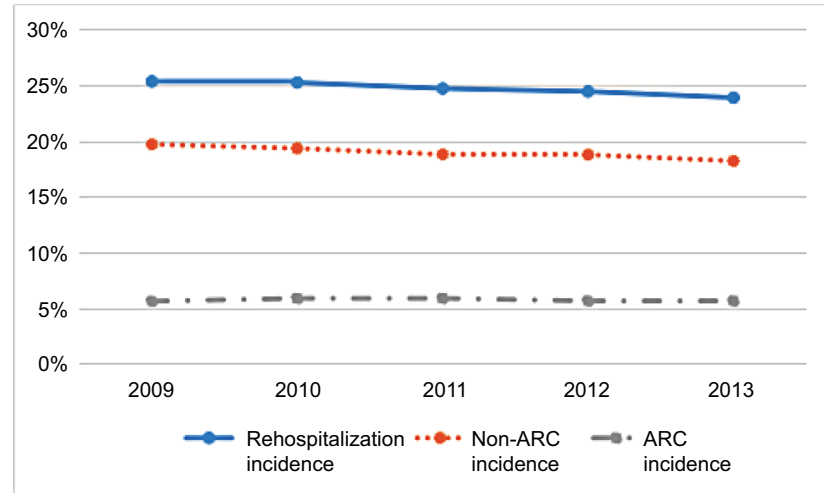

Figure I Trend in rehospitalization, non-ARC, and ARC incidence I year post colectomy. ${ }^{\mathrm{a}}$

Note: ${ }^{a}$ Non-ARC and ARC are classified based on the first rehospitalization event. Abbreviation: ARC, adhesion-related complication. compared to non-ARC (28.6\%) and ARC (21.8\%) patients. ARC patients had a higher rate of multiple procedures and adhesiolysis (37.3\% and $0.5 \%$, respectively) vs non-ARC patients (32.9\% and $0.2 \%$, respectively).

\section{Resource utilization of ARC and non- ARC readmissions}

Table 4 presents the resource utilization of ARC and nonARC events. Among patients who had an ARC event, mean LOS was 7.2 days ( $\mathrm{SD}=8.0$, median=5.0 days) and mean total reimbursement was $\$ 29,802$ ( $\mathrm{SD}=\$ 43,037$, median $=\$ 18,823)$. For non-ARC events, the mean LOS was 5.2 days $(\mathrm{SD}=6.8$, median=3.0 days) and mean total cost was $\$ 22,476(\mathrm{SD}=\$ 36,120$, median $=\$ 14,106)$. ARC events had a higher MCC MS-DRG proportion compared to non-ARC events: $40.4 \%$ vs $34.2 \%$, respectively. CT imaging at index was also higher among those who were readmitted for ARC $(24.8 \%)$ vs non-ARC (20.5\%).

\section{Risk factors associated with ARC}

A total of 64,532 patients, of whom 3,704 had ARC events were included in the multivariable logistic regression models. Patient and procedure characteristics from Tables 1 and 2 were included in multivariable logistic regression models as covariates. Table 5 and Figure 2 represent the significant risk factors associated with ARC within 1 year of readmission. Adhesiolysis at index was associated with a significantly higher odds of ARC (OR=2.45, 95\%

Table 4 Resource utilization for first postcolectomy rehospitalization

\begin{tabular}{|c|c|c|c|}
\hline & Non-ARC rehospitalization & ARC rehospitalization & $P$-value \\
\hline $\mathrm{n}$ & 12,229 & 3,704 & \\
\hline \multicolumn{4}{|l|}{ Time to rehospitalization (days) } \\
\hline Mean & 137 & 132 & $<0.000 \mathrm{I}^{\mathrm{a}}$ \\
\hline Median & 113 & 112 & \\
\hline SD & 106 & 101 & \\
\hline \multicolumn{4}{|c|}{ Total rehospitalization cost (2015 USD) } \\
\hline Mean & 22,476 & 29,802 & $<0.000 \mathrm{I}^{\mathrm{a}}$ \\
\hline Median & 14,106 & 18,823 & \\
\hline SD & 36,130 & 43,037 & \\
\hline \multicolumn{4}{|l|}{ Rehospitalization LOS (days) } \\
\hline Mean & 5.2 & 7.2 & $<0.000 I^{a}$ \\
\hline Median & 3.0 & 5.0 & \\
\hline SD & 6.8 & 8.0 & \\
\hline MS-DRG category & & & $<0.00 I^{b}$ \\
\hline $\mathrm{CC}$ & $45.4 \%$ & $40.5 \%$ & \\
\hline MCC & $34.2 \%$ & $40.4 \%$ & \\
\hline W/o MCC/CC & $20.4 \%$ & $19.2 \%$ & \\
\hline CT scan (any time post index) & $42.0 \%$ & $51.6 \%$ & $0.0482^{\mathrm{b}}$ \\
\hline
\end{tabular}

Notes: aWilcoxon signed-rank test. ${ }^{b} \mathrm{Chi}$-square test.

Abbreviations: ARC, adhesion-related complication; 2015 USD, 2015 US dollars; LOS, length of stay; MS-DRG, Medicare Severity Diagnosis Related Groupings; CC, complication or comorbidity; MCC, major complication or comorbidity; w/o, without; CT, computed tomography. 
Table 5 Significant risk factors related to ARC within I year

\begin{tabular}{|c|c|c|c|c|}
\hline Risk factors & OR & $\begin{array}{l}95 \% \\
\text { LCL }\end{array}$ & $\begin{array}{l}95 \% \\
\text { UCL }\end{array}$ & $P$-value \\
\hline \multicolumn{5}{|l|}{ Patient demographics } \\
\hline Age (years): $45-54$ vs $18-44$ & 0.90 & 0.81 & 1.00 & 0.050 \\
\hline Age (years): $55-64$ vs $18-44$ & 0.90 & 0.81 & 1.00 & 0.040 \\
\hline Age (years): $75+$ vs $18-44$ & 0.52 & 0.3 & 0.88 & 0.010 \\
\hline Indication: other vs cancer & 1.12 & 1.02 & 1.22 & 0.017 \\
\hline Indication: IBD vs cancer & 1.69 & 1.43 & 1.99 & $<0.000$ I \\
\hline \multicolumn{5}{|l|}{ Patient comorbidities } \\
\hline Obesity $^{\mathrm{a}}$ & 0.87 & 0.76 & 0.99 & 0.040 \\
\hline Hypertension uncomplicated & 0.86 & 0.79 & 0.92 & $<0.0001$ \\
\hline Peripheral vascular disorders ${ }^{a}$ & 1.23 & 1.05 & 1.44 & 0.012 \\
\hline Hypertension complicated ${ }^{a}$ & 1.28 & 1.05 & 1.56 & 0.015 \\
\hline Fluid and electrolyte disorders ${ }^{\mathrm{a}}$ & 1.36 & 1.24 & 1.49 & $<0.0001$ \\
\hline Weight loss ${ }^{\mathrm{a}}$ & 1.48 & 1.3 & 1.68 & $<0.000$ I \\
\hline \multicolumn{5}{|l|}{ Procedural characteristics } \\
\hline Approach: lap vs open & 0.42 & 0.39 & 0.46 & $<0.0001$ \\
\hline Resection: partial vs right & 1.27 & 1.05 & 1.44 & 0.012 \\
\hline Resection: left vs right & 1.69 & 1.53 & 1.86 & $<0.0001$ \\
\hline Resection: total vs right & 3.77 & 3.27 & 4.36 & $<0.0001$ \\
\hline Number of procedures (I vs $2+$ ) & 1.38 & 1.29 & 1.49 & $<0.0001$ \\
\hline $\mathrm{CT}$ imaging at index & 1.79 & 1.65 & 1.95 & $<0.0001$ \\
\hline Adhesions at index & 2.45 & 1.42 & 4.23 & 0.0013 \\
\hline Year: 2010 vs 2009 & 1.28 & 1.15 & 1.43 & $<0.000$ I \\
\hline Year: 20II vs 2009 & 1.41 & 1.26 & 1.57 & $<0.0001$ \\
\hline Year: 2012 vs 2009 & 1.40 & 1.24 & 1.57 & $<0.0001$ \\
\hline Year: 2013 vs 2009 & 1.40 & 1.25 & 1.58 & $<0.0001$ \\
\hline
\end{tabular}

Notes: aElixhauser comorbidities.

Abbreviations: ARC, adhesion-related complication; LCL, lower confidence limit; UCL, upper confidence limit; IBD, inflammatory bowel disease; CT, computed tomography.
CI [1.42-4.23]). Resection type was also associated with increased odds of ARC (compared to right-sided resections): total colectomy ( $\mathrm{OR}=3.77,95 \% \mathrm{CI}[3.27-4.36])$, left-sided resections (OR=1.69, 95\% CI [1.53-1.86]), and partial colectomy $(\mathrm{OR}=1.27,95 \% \mathrm{CI}[1.14-1.41])$. Other factors that increased odds of ARC included CT imaging at index (OR=1.79, 95\% CI [1.65-1.95]), surgical indication: IBD vs cancer $(\mathrm{OR}=1.69,95 \% \mathrm{CI}[1.43-1.99])$, and multiple abdominal procedures at index $(\mathrm{OR}=1.38,95 \% \mathrm{CI}$ [1.29-1.49]). Intraoperative risk factors such as laparoscopic approach at index had a protective effect with $68 \%$ lower odds of an ARC compared to open surgery ( $\mathrm{OR}=0.42,95 \%$ CI [0.39-0.46]).

\section{Discussion}

The present study explored ARC within 1 year after colon resections. ARC incidence, time to event, risk factors and healthcare utilization for inpatient hospitalizations, and costs were estimated. In the present study, ARC were defined broadly as diagnosis of ileus, $\mathrm{SBO}$, or an adhesiolysis procedure occurring during the first readmission event after colon resections. In our study, $\sim 26 \%$ of colon resection patients had a readmission within the first year with $20 \%$ non-ARC and $5.7 \%$ ARC related and varied according to type of resection from $19.3 \%$ for total colectomy to $4.1 \%$ for right colon



Figure 2 Significant risk factors related to ARC within I year after colectomy.

Note: *Elixhauser comorbidities.

Abbreviations: ARC, adhesion-related complication; CT, computed tomography; IBD, inflammatory bowel disease. 
resection. The incidences for the three individual nonmutually exclusive components of ARC were $2.7 \%$ for SBO, $2.0 \%$ for ileus, and $1.7 \%$ for adhesiolysis.

There is a substantial heterogeneity in the published literature regarding the definition, the type of abdominal surgeries evaluated, and time period of evaluation for complications related to adhesions. Other studies have estimated the incidence of adhesion complications for a variety of abdominal procedures. A large study from Scotland (Surgical and Clinical Adhesions Research [SCAR] study) followed 12,584 patients who underwent open abdominal surgery (small intestine, appendix, colon, rectum, and abdominal wall) in 1986. Postoperative adhesions were defined as a clinical decision regarding adhesion readmissions and reoperations. Over a 10 -year period, the average readmission rate for the colon group ranged from $8.2 \%$ to $68.9 \%$ depending on the clinical probability of the adhesion diagnosis (directly, possibly, or potentially related). ${ }^{14}$ In follow-up analysis (SCAR-2), a more recent set of open colorectal surgery patients (1996-1999) were evaluated utilizing the same clinical definition for adhesion complications. The yearly readmission rate for adhesion complications was $9.0 \%, 13.6 \%, 17.0 \%$, and $19.0 \%$ for years 1 through 4 , respectively (directly or possibly related). ${ }^{15}$ The most recent SCAR-3 study estimated the 5-year incidence for adhesion complications for specific colon resections (incidence, total number of patients): total colectomy $(8.8 \%$, $160)$, right hemicolectomy $(3.8 \%, 1,235)$, left hemicolectomy $(4.9 \%, 1,023)$, and sigmoidectomy $(4.7,779) .{ }^{16}$ Another study estimated the incidence of adhesion-related bowel obstruction in laparoscopic colorectal surgery at $2.0 \%$ (though the incidence of any bowel obstruction was $4.2 \%$ ) with an intent to treat analysis with mean follow-up of 38 months. ${ }^{17}$

Risk factors identified in this study included resection type (total colectomy and left-sided colectomy were associated with increased odds for ARC compared to right colon resections), adhesiolysis at index, number of abdominal procedures at index, younger age, and indication for surgery with IBD having higher odds compared to cancer. Total colectomy and left colon procedures generally have increased complexity and more extensive tissue dissections compared to right colon resections. Similarly, the more abdominal procedures performed increases the tissue dissection and likely contributes to increased scar formation. CT scan at index was also a risk factor and could represent increased surgical complexity or a more complex surgical presentation. Patients who require adhesiolysis at index have demonstrated a history of adhesion formation, which has been shown to be a risk factor for future ARCs. Laparoscopic approach was associated with a $58 \%$ reduction in odds for ARCs. These risk factors are in general similar to risk factors identified in previous clinical studies.

In our study, other identified factors not previously evaluated or identified in the literature included patient comorbidities measured using the Elixhauser comorbidities: weight loss, fluid and electrolyte disorders, and peripheral vascular disorders. Obesity and uncomplicated hypertension had a protective effect on ARC; it is unclear what clinical implication these may have given the potential for confounding or other biases.

The SCAR-3 study includes a univariate analysis of the adhesion complication incidence and found that younger age ( $<60$ years), previous abdominal surgery, and type of resection (total colectomy the highest and right colectomy) had higher rates of adhesion complications. ${ }^{16} \mathrm{~A}$ prospective analysis focusing on recurrent adhesion complications (both obstruction and ileus) for multiple types of initial adhesive bowel obstruction procedures utilized Kaplan-Meier cumulative incidence and estimated 1-year incidence of 5.5\% (95\% CI [3.1-6.9]). Significant risk factors identified from the Cox regression included younger age $(<40$ years compared to $>75$ years, HR 2.70 [95\% CI $\{1.48-5.94\}$ ) and presence of obstructive or matted adhesions vs simple bands at index operation (HR 3.79 [95\% CI $\{1.84-7.78\}$ ). Other factors evaluated but not significantly included were laparoscopic vs open approach, American Society of Anesthesia score, number and site of previous operations, and postoperative surgical and medical conditions. ${ }^{18}$ Multiple studies have found decreased adhesion complications associated with laparoscopic compared to open surgical approach. Stommel et $\mathrm{a}^{20}$ reported a lower incidence, extent, and severity of adhesions to parietal surfaces after laparoscopic colon resection compared to open approach $(37.7 \%$ vs $78.9 \% ; P<0.001)$. A literature and meta-analysis estimated the incidence of SBO for any cause after abdominal surgery at $9 \%(95 \%$ CI [7-10], $\left.I^{2}=99 \%\right)$ and adhesive $\mathrm{SBO}$ at $2 \%\left(95 \% \mathrm{CI}[2-3], I^{2}=93 \%\right)$. The incidence differed according to surgical approach with laparoscopy, 2.7\% (95\% CI [2.3-3.1\%]), having a lower incidence compared to laparotomy, $3.8 \%(95 \% \mathrm{CI}[3.1-4.4]){ }^{7}$ Another meta-analysis evaluated the risk ratios for adhesive SBO between laparoscopic and open surgery for both randomized trials, $0.26\left(95 \% \mathrm{CI}[0.10-0.67], P^{2}=41 \%\right)$, and nonrandomized studies, $0.56\left(95 \% \mathrm{CI}[0.06-0.96], I^{2}=91 \%\right){ }^{4}$

Our study quantified the resource utilization of ARC events and contextualized them with other first-time rehospitalization episodes occurring the first year postcolon surgery. In the present study, the mean hospital LOS among the ARC 
cohort was 7 days with associated average reimbursement per episode of $\$ 29,000$, which was more than that for non-ARC rehospitalizations $(\$ 22,476)$. Krielen et $\mathrm{al}^{21}$ reported in-hospital costs associated with treatment of adhesive SBO using data on patients admitted with adhesive SBO to the Radboud University Medical Center (the Netherlands) between November 2013 and November 2015 (number of admissions=39). Overall cost for an admission for adhesive SBO with operative treatment was $€ 16,305$ ( $\mathrm{SD} € 2,513$ ) and for nonoperative treatment was $€ 2,277$ ( $\mathrm{SD} € 265 ; P \leq 0.001$ ). Using the correction coefficients and conversion rates, these costs translate into $\sim \$ 21,229$ for operative treatment and $\$ 2,964$ (USD) for nonoperative treatment. Our study did not evaluate operative vs nonoperative treatment for either ARC or non-ARC readmissions.

Limitations of this study are inherent to utilizing administrative claims databases for clinical research. Specific clinical variables such as the extent of colon pathology, comorbidities, or precise surgical resection technique are not available. There may also be misclassification of both diagnosis codes utilized for covariates and procedures. The ARC end point was a combination of both SBO and ileus diagnoses and adhesiolysis occurring during the first rehospitalization event within a year of the index surgery. Some of these readmissions while associated with an adhesionrelated code may not be causally related to adhesions, and the extent of an alternative nonadhesion cause is unknown. For example, a planned colostomy reversal with an adhesiolysis may indicate either a more complex procedure or an incidental finding, rather than a complication. ARC was defined as the first inpatient rehospitalization within a year postcolon resection and does not include ARC events that occurred after the first inpatient readmission, and this may underrepresent the incidence of ARC. Because continuous enrollment was required for 1 year postindex, colectomy patients who had an ARC and subsequently died within the first year or lost eligibility due to adhesion complications would not have been included in the analysis. While this is assumed to be a small number, the costs related to these events may be substantial and their exclusion may bias the hospitalization costs. Outcomes were limited to LOS and rehospitalization costs and did not include other events such as emergency room, rehabilitation, and home health. Medical costs reported may be underrepresented due to the $65+$ age categories containing only Medicare supplemental patients in Truven database. Finally, a detailed analysis on ARC resource utilization and clinical outcomes differences between surgical vs conservative therapy for ARC was not performed and is an important area for future research.
Despite the clinical importance in terms of incidence, morbidity, and resource utilization, a study found that out of 200 patients who were to undergo abdominal surgery, only $8.5 \%$ had either adhesions or ARCs (bowel obstruction, need for further surgery, pain, or infertility) on their consent forms. ${ }^{19}$ This potentially points to a disconnect between the surgical reality and communication to the patients and illustrates the need for our relevant and timely research. The strength of this study is that we utilized a large hospital database that captures the timing and actual reimbursement costs for ARC along with a large number of patients to allow for evaluation of procedural risk factors.

\section{Conclusion}

ARCs occurred in 5.7\% of all patients and accounted for almost one-quarter of all first inpatient readmissions within the first year after colon resection. These rehospitalization events have substantial healthcare utilization in terms of length of hospitalization and costs. Risk factors for ARC include indication and type of colon surgery and indicators for more complex index surgeries (such as multiple procedures and adhesiolysis). The laparoscopic surgical approach was associated with a reduction in the odds of ARC. These factors are important to consider for patient communication of these potential complications along with economic and clinical outcome performance risk adjustment.

\section{Acknowledgments}

The study was funded by Johnson \& Johnson. The abstract of this paper was presented at the 19th Annual ISPOR EU - "Rate and Healthcare Utilization (LOS and Costs) of AdhesionRelated Complications after Colon Resection Procedures" as a poster presentation with interim findings. The poster's abstract was published in "Poster Abstracts" in Value in Health (https://www.valueinhealthjournal.com/article/S1098$\underline{3015(16) 32315-4 / \text { abstract)} \text {. }}$

\section{Disclosure}

All authors are employees of Johnson \& Johnson. The authors report no other conflicts of interest in this work.

\section{References}

1. Decherney AH, Dizerega GS. Clinical problem of intraperitoneal postsurgical adhesion formation following general surgery and the use of adhesion prevention barriers. Surg Clin North Am. 1997;77(3):671-688.

2. González-Quintero VH, Cruz-Pachano FE. Preventing adhesions in obstetric and gynecologic surgical procedures. Rev Obstet Gynecol. 2009;2(1):38-45.

3. Sikirica V1, Bapat B, Candrilli SD, Davis KL, Wilson M, Johns A. The inpatient burden of abdominal and gynecological adhesiolysis in the US. BMC Surg. 2011;9(11):13. 
4. Ha GW, Lee MR, Kim JH. Adhesive small bowel obstruction after laparoscopic and open colorectal surgery: a systematic review and meta-analysis. Am J Surg. 2016;212(3):527-536.

5. Ellis H, Moran BJ, Thompson JN, et al. Adhesion-related hospital readmissions after abdominal and pelvic surgery: a retrospective cohort study. Lancet. 1999;353(9163):1476-1480.

6. Scott JW, Olufajo OA, Brat GA, et al. Use of national burden to define operative emergency general surgery. JAMA Surg. 2016;151(6):e160480.

7. Ten Broek RPG, Issa Y, van Santbrink EJP, et al. Burden of adhesions in abdominal and pelvic surgery: systematic review and met-analysis. BMJ. 2013;347(oct03 1):f5588.

8. Tulandi T, Al-Jaroudi D. Nonclosure of peritoneum: a reappraisal. Am J Obstet Gynecol. 2003;189(2):609-612.

9. Ouaïssi M, Gaujoux S, Veyrie N, et al. Post-operative adhesions after digestive surgery: their incidence and prevention: review of the literature. J Visc Surg. 2012;149(2):104-114.

10. Wick EC, Shore AD, Hirose K, et al. Readmission rates and cost following colorectal surgery. Dis Colon Rectum. 2011;54(12):1475-1479.

11. Smolarek S, Shalaby M, Paolo Angelucci G, et al. Small-bowel obstruction secondary to adhesions after open or laparoscopic colorectal surgery. JSLS. 2016;20(4)e2016.00073.

12. Sileri P, Sthory R, Mcveigh E, et al. Adhesions are common and costly after open pouch surgery. J Gastrointest Surg. 2008;12(7):1239-1245.

13. Elixhauser A, Steiner C, Harris DR, Coffey RM. Comorbidity measures for use with administrative data. Med Care. 1998;36(1):8-27.
14. Parker MC, Ellis H, Moran BJ, et al. Postoperative adhesions: ten-year follow-up of 12,584 patients undergoing lower abdominal surgery. Dis Colon Rectum. 2001;44(6):822-829.

15. Parker MC, Wilson MS, Menzies D, et al. Colorectal surgery: the risk and the rate of subsequent surgery for adhesive SBO. Colorectal Dis. 2004;6:506-511.

16. Parker MC, Wilson MS, Menzies D, et al. The SCAR-3 study: 5-year adhesion-related readmission risk following lower abdominal surgical procedures. Colorectal Dis. 2005;7(6):551-558.

17. Rosin D, Zmora O, Hoffman A, et al. Low incidence of adhesion-related bowel obstruction after laparoscopic colorectal surgery. J Laparoendosc Adv Surg Tech A. 2007;17(5):604-607.

18. Duron JJ, Silva NJ, du Montcel ST, et al. Adhesive postoperative small bowel obstruction: incidence and risk factors of recurrence after surgical treatment: a multicenter prospective study. Ann Surg. 2006;244(5):750-757.

19. Rajab TK, Wallwiener M, Talukdar S, Kraemer B. Adhesion-related complications are common, but rarely discussed in preoperative consent: a multicenter study. World J Surg. 2009;33(4):748-750.

20. Stommel MWJ, Ten Broek RPG, Strik C, et al. Multicenter observational study of adhesion formation after open-and laparoscopic surgery for colorectal cancer. Ann Surg. 2018;267(4):743-748.

21. Krielen P, van den Beukel BA, Stommel MWJ, van Goor H, Strik C, Ten Broek RPG. In-hospital costs of an admission for adhesive small bowel obstruction. World J Emerg Surg. 2016;11:49. 


\section{Supplementary material}

Table SI Diagnosis and procedure codes for ARC events

\begin{tabular}{llll}
\hline Data type & Code & Definition & Anatomy \\
\hline ICD-9 & 560.1 & Paralytic ileus & Ileus \\
ICD-9 & 560.3 & Impaction of intestine, unspecified & Impaction \\
ICD-9 & 560.39 & Other impaction of intestine & Impaction \\
ICD-9 & 560.81 & Intestinal or peritoneal adhesions with obstruction (postoperative, postinfection) & Obstruction \\
ICD-9 & 560.89 & Other specified intestinal obstruction & Obstruction \\
ICD-9 & 560.9 & Unspecified intestinal obstruction & Obstruction \\
ICD-9 & 54.51 & Laparoscopic lysis of peritoneal adhesions & Lysis of adhesions \\
ICD-9 & 54.59 & Other lysis of peritoneal adhesions & Lysis of adhesions \\
\hline
\end{tabular}

Note: Must occur with first inpatient readmission.

Abbreviations: ARC, adhesion-related complication; ICD-9, ICD ninth revision.

ClinicoEconomics and Outcomes Research is an international, peerreviewed open-access journal focusing on health technology assessment, pharmacoeconomics and outcomes research in the areas of diagnosis, medical devices, and clinical, surgical and pharmacological intervention. The economic impact of health policy and health systems organization also constitute important areas of coverage. The manuscript management system is completely online and includes a very quick and fair peer-review system, which is all easy to use. Visit http://www.dovepress.com/testimonials.php to read real quotes from published authors. 